\title{
Processo de implementação de um sistema de gestão de custos em hospital público: um estudo das variáveis intervenientes
}

The implementation process of a cost management system into a public hospital: a study of the intervening factors

\author{
Altair Borgert \\ Doutor em Engenharia de Produção na Universidade Federal de Santa Catarina \\ Professor do Programa de Pós-Graduação em Contabilidade da Universidade Federal de \\ Santa Catarina \\ Endereço: Campus Universitário, Bairro Trindade \\ CEP: 88040-970 - Florianópolis/SC - Brasil \\ E-mail: altair@borgert.com.br \\ Telefone: (48) 3721-9383
}

\section{Rodrigo Viega Alves}

Mestre em Administração na Universidade Federal de Santa Catarina

Endereço: Rua Fabrício Pilar, 810, Ap. 306 - Mont Serrat

CEP: 90450-040 - Porto Alegre/RS - Brasil.

E-mail: rodrigovie@hotmail.com

\section{Charles Albino Schultz}

Mestre em Contabilidade na Universidade Federal de Santa Catarina

Doutorando em Wirtschaftswissenschaften na Technische Universität Chemnitz

Professor do curso de Administração da Universidade Federal da Fronteira Sul

Endereço: UFFS - Unidade Seminário, Acesso Canários da Terra, S/N, Bairro Seminário, Caixa Postal 181

CEP: 89812-000 - Chapecó/SC - Brasil

E-mail: charles_mcr@yahoo.com.br

Telefone: (49) 2049-1500 


\title{
Resumo
}

O presente artigo analisa fatores intervenientes no processo de implementação de um sistema de gestão de custos em um hospital público do Rio Grande do Sul, Brasil. Após a identificação de dez fatores na literatura, realizou-se um estudo empírico junto aos funcionários do setor de ortopedia na busca da percepção de dificuldades em relação aos fatores identificados. Concluiu-se que a falta de planejamento do projeto é considerada a maior dificuldade enquanto que a utilização de softwares foi considerada menos importante. Ainda, com base nas informações obtidas com os gestores constataram-se problemas de comunicação entre os setores administrativo e ortopedia.

Palavras-chave: Sistema de gestão de custos. Gestão hospitalar. Custos hospitalares.

\begin{abstract}
This paper analyzes intervening factors in the process of implementation of a cost management system into a public hospital of the state of Rio Grande do Sul, Brazil. After the identification of ten factors in the literature it has been developed an empiric study with the employees from the orthopedics area in order to find out the employees' perception of difficulties in relation to the identified factors. The conclusion is that the lack of planning the project is the biggest difficulty while the use of software was considered as the less important one. And, based on the information obtained with the managers, we noticed that there are not enough communication between the administrative director and the area director.
\end{abstract}

Keywords: Cost management system. Hospital management. Hospital costs.

\section{Introdução}

A incorporação de características empresariais tem modificado o ambiente hospitalar nas últimas décadas, de modo que o processo produtivo se torna mais voltado ao cliente para que seja possível, cada vez mais, alcançar melhores níveis de produtividade.

Com esta mudança, informações gerenciais se tornam necessárias, dentre as quais as de custos, que assumem papel de destaque nas organizações hospitalares. Assim, um sistema de gestão de informações torna-se um importante fator para que a prestação dos serviços de atenção e pesquisa em saúde atinja alto nível de eficiência, bem como alcance um bom desempenho econômico e financeiro (WINTER; HAUX, 1994). A coleta dos dados e o processamento destas informações fazem parte de uma parcela do trabalho dos profissionais de um hospital, sejam eles médicos, enfermeiros ou administradores.

No momento da escolha de um sistema de gestão de custos deve-se considerar o conjunto de dificuldades que ocorrem no processo de implementação deste. Estes fatores são obstáculos que, muitas vezes, levam hospitais a desistirem da implementação do sistema ou a subutilizá-los após a sua implementação (ROCHA, 2004).

Os gastos mundiais com saúde vêm crescendo nas últimas décadas e, de acordo com Lima e Lima (1998), muitos recursos têm sido desperdiçados com 
o mau uso de tecnologias médicas; subutilização da capacidade física instalada; má distribuição de pessoal; e, com aquisição de produtos farmacêuticos de marca registrada ao invés de genéricos.

No Brasil ocorreram mudanças importantes no setor hospitalar com o advento da Lei 9.656 (BRASIL, 1998) e a criação da Agência Nacional de Saúde Suplementar (PATTIO, 2001). Com isso, criou-se um maior número de regulamentações e novas exigências, e garantias para o consumidor. Mas, por outro lado, isto gerou um aumento nos custos das operadoras dos planos de saúde que, por sua vez, repassaram parte destes custos para os prestadores de serviços de saúde o que, conseqüentemente, acarretou um aumento nos custos do sistema público de saúde. Segundo Carpintéro (1999), nos últimos anos as referências sobre custos na área da saúde se intensificaram justamente devido ao aumento nos gastos públicos e à maior importância dada ao gerenciamento dos custos do setor da saúde.

Observa-se que os estudos sobre custos na área da saúde, publicados em congressos e periódicos, além de serem em quantidades modestas, normalmente, se constituem de estudos voltados a levantamentos de custos, e testes de adaptabilidade de determinados sistemas e métodos de custeio. Poucos são os estudos que abordaram os fatores que influenciam o sucesso na implementação e manutenção de um sistema empresarial em um hospital. Dentre os motivos para esta situação pode-se citar a complexidade e a dificuldade de acesso ao ambiente hospitalar. Assim, considerando a escassez de estudos nesta área, justifica-se a realização deste estudo investigativo sobre as dificuldades e facilidades que intervém na implementação de sistemas de gestão de custos em hospitais.

Portanto, o objetivo geral deste trabalho consiste na identificação dos fatores que influenciam o processo de implementação de um sistema de gestão de custos em um hospital público do Rio Grande do Sul. De uma forma mais específica o estudo busca responder as seguintes questões: a) quais os fatores presentes no processo de implementação de um sistema de gestão de custos? b) quais as principais dificuldades percebidas pelos profissionais de saúde de diferentes níveis? c) quais as principais dificuldades na percepção dos gestores do hospital?

\section{Metodologia}

Esta pesquisa se caracteriza como um estudo descritivo que, conforme Selltiz, Wrightsman e Cook (1987), apresenta as particularidades de uma situação, grupo de indivíduos ou indivíduo. Além disso, pode determinar a freqüência com que um fenômeno ocorre. Quanto à forma de abordagem do problema, pode-se classificá-la como quantitativa, uma vez que é orientada à identificação das dificuldades ou facilidades percebidos pelos profissionais de saúde no processo de 
implementação de um sistema de custos no contexto da instituição hospitalar.

Como a pesquisa se desenvolve em um hospital, esta também se caracteriza como estudo de caso. Para Gil (1992), o estudo de caso é caracterizado pelo estudo profundo e exaustivo de um ou de poucos objetos, de maneira a permitir conhecimento amplo e detalhado do mesmo. Assim, deseja-se buscar explicações para o caso específico, já que os participantes da pesquisa são profissionais da saúde do setor de ortopedia, bem como gestores gerais de um hospital público do estado do Rio Grande do Sul.

Por acessibilidade, a escolha do caso recai sobre uma instituição pública que já possui um sistema de custos implementado. Porém, o foco no setor de ortopedia do hospital se deve ao fato de se tratar de um setor de referência no Estado do Rio Grande do Sul e, também, por possuir 102 funcionários. Contudo, por questões éticas, preserva-se a identidade do próprio hospital, bem como das pessoas envolvidas.

O grupo de entrevistados é formado por médicos, auxiliares e técnicos em enfermagem, técnicos gessistas, enfermeiros e fisioterapeutas. A amostra compreende 66 funcionários, o que representa $64,7 \%$ do total do setor, uma vez que alguns estão cedidos para outros setores e, ainda, alguns que optaram por não participar da pesquisa e/ou não devolveram o questionário.

Neste sentido, a pesquisa se deu por meio da aplicação de entrevistas do tipo não-estruturada com os gestores administrativos do hospital e com a aplicação dos questionários - com questões objetivas e abertas - aos profissionais da saúde. Com base nos questionários e nas entrevistas, e com base nos tópicos levantados na revisão bibliográfica, identificaram-se as principais facilidades e dificuldades na implementação do sistema de gestão de custos. A definição por este método de coleta de dados se deve ao fato de o questionário cumprir pelo menos duas funções importantes: descrever características e medir determinadas variáveis de um grupo social. Já a entrevista é uma técnica que permite o estreitamento da relação entre pessoas (RICHARDSON, 1989). Assim, os dados levantados por meio de questionários com os funcionários, juntamente com as entrevistas dos gestores, serviram para a identificação de atividades e processos, como também, para um melhor entendimento do ambiente hospitalar.

Por se tratar de uma pesquisa social que envolve o comportamento humano, o estudo enfrenta limitações, devendo-se, neste caso, observar atentamente as respostas obtidas, uma vez que, nem sempre, os entrevistados apresentam a mesma predisposição para participar de pesquisas. Já, pela característica de estudo de caso, Gil (1992) explicita que os resultados obtidos neste tipo de pesquisa não permitem sua generalização. Outra limitação decorre do fato do hospital exigir sigilo em alguns aspectos, o que dificulta uma análise mais profunda do caso. Uma última limitação diz respeito à inferência do(s) pesquisador(es) na interpretação 
dos questionários e entrevistas, bem como nas conclusões e observações finais.

Um aspecto importante do estudo é a observação de que alguns pesquisados não responderam todas as questões formuladas, e outros, por sua vez, marcaram mais de uma alternativa por entenderem que ambas sejam corretas para o seu caso. Assim, nas análises deve-se observar que o número de respostas não é igual em todas as questões, porém, estas diferenças não são suficientemente significativas para interferirem nas conclusões do estudo.

\section{Base Teórica para a Formulação dos Questionários e Entrevistas}

A dificuldade no processo de implementação de sistemas de custos em instituições de saúde decorre, em parte, da grande diversidade de serviços prestados nos hospitais e da complexa composição destes serviços (CARPINTÉRO, 1999).

Antes do início do processo de implementação de um sistema de custos, devem-se analisar os diversos fatores envolvidos, dentre os quais as características culturais da instituição, os objetivos da implementação do sistema e os recursos financeiros disponíveis.

Alguns destes fatores que influenciam a implementação de sistemas de gestão de custos, identificados durante a revisão bibliográfica são apresentados na sequência.

3.1 Divisão entre a Área Administrativa e a da Saúde, e a Participação da Direção

A participação da direção no processo de implementação de um sistema traduz aos demais funcionários a importância do projeto para a instituição. Cabe à direção mostrar as vantagens das ferramentas gerenciais que podem ser utilizadas, inclusive para facilitar a atuação do profissional da saúde em seus processos operacionais. Também, devem ser envolvidos no projeto todos os responsáveis pelos setores. Segundo Panegalli (2004), para que uma entidade seja bem sucedida é necessário que seus integrantes tenham uma visão orgânica comum dos principais objetivos, metas e recursos disponíveis, bem como a aceitação do planejamento estratégico, operacional e organizacional.

Como, normalmente, há uma dicotomia entre a área administrativa e a área da saúde, é necessário que os gestores se empenhem no envolvimento e comprometimento de todos com o sistema, iniciando pelos diretos da área clínica. Quando não há a participação destes diretores cria-se uma imagem entre os demais profissionais da saúde de que se trata de uma ação estritamente administrativa (ROCHA, 2004) e, portanto, estão livres de qualquer responsabilidade. 
Como o sistema de custos envolve diversos setores interligados, também se torna o envolvimento das pessoas destes setores essencial para o planejamento do projeto, principalmente aquelas envolvidas diretamente na implementação. Rodrigues Filho, Xavier e Adriano (2001) apontam a não inclusão dos usuários, principalmente dos profissionais médicos, no desenho e na implementação dos sistemas de informações hospitalares, como responsável por grande parte da falta de sucesso destes sistemas.

\subsection{Falta de Conhecimento para a Utilização das Informações}

As informações de custos geradas pelo sistema são essenciais para diversas áreas, dentre as quais se podem enumerar a do orçamento, do controle e da redução de custos, da avaliação de desempenho, do planejamento e da tomada de decisões econômicas (COSTA; CARVALHO, 2007). Como muitos administradores hospitalares não possuem conhecimento da área administrativa o grau de dificuldade para a utilização das informações de custos é maior. Algumas vezes, é assumida a crença de que após a implementação do sistema tudo o que está relacionado com os custos estará resolvido quando, na verdade, o sistema em si nada faz. Cabe às pessoas interpretarem as informações e tomarem as medidas necessárias.

O fato de tais gestores não possuírem conhecimentos para a utilização das informações geradas por um sistema de custos contribui, ainda, para que fiquem com receio dos sistemas de controle, pois desconhecem as variáveis do sistema a ser implantado. Assim, a falta de conhecimento dos gestores deve ser considerada, o que reforça novamente a necessidade da integração destes no processo, desde as fases iniciais, para que estes tenham a possibilidade de, gradativamente, entender o objetivo, a estrutura e a importância do sistema, bem como a utilidade e as formas de utilização das informações disponíveis.

\subsection{Resistência Cultural}

Outro fator que se apresenta no processo de implementação de um sistema de custos é a dificuldade que os profissionais da área da saúde têm em se adaptar à utilização dos sistemas de controle. É preciso que o sistema de custos seja adaptado, o máximo possível, à cultura da organização hospitalar para que este possa ser, mais facilmente, legitimado (ALVES; BORGERT; SCHULTZ, 2006). Portanto, é importante que o controle seja incorporado na cultura da organização.

Segundo Salgues et al (2005), a cultura organizacional é um conjunto de valores e experiências compartilhadas que vão sendo aceitas pelos membros do grupo, se cristalizam no inconsciente deste e, posteriormente, começam a guiar o comportamento do grupo. Assim, é necessário que o controle do sistema de custos 
e o próprio sistema sejam introduzidos na cultura hospitalar vigente.

A resistência cultural tem se mostrado uma das principais dificuldades no processo de implementação de um sistema de custos, principalmente porque os funcionários não querem mudar a sua rotina diária e têm receio da competição interna entre as unidades do hospital ou com o colega de profissão. Também, têm receio de que, com a aplicação do sistema de custos, a instituição possa concluir que o seu trabalho é ineficiente (ALVES; BORGERT; SCHULTZ, 2006).

Porém, diversas vezes a resistência cultural decorre da falta de divulgação apropriada dos objetivos da implantação do sistema de custos, ou pela falta de introdução dos novos conceitos para o entendimento das novas condutas requeridas (POMPERMAYER, 1999).

Em outro estudo, realizado no estado do Rio Grande do Sul, Borba et al (2003) detectaram que a dificuldade mais assinalada pelos hospitais foram as relacionadas ao processo de mudança organizacional, que envolvem mudança da cultura, resistência ao uso do sistema e necessidade de treinamento dos funcionários.

\subsection{Treinamento}

$\mathrm{Na}$ maioria dos processos realizados nas organizações hospitalares estão envolvidos profissionais da área da saúde, o paciente e a restauração da sua saúde, que estão em primeiro lugar na escala de importância, o que faz com que custos sejam fatores secundários. Cabe aos responsáveis pela implementação do sistema agir como facilitadores no processo, de forma que durante o levantamento das atividades os diversos colaboradores sejam envolvidos (ROCHA, 2004). Desta forma cria-se, também, um envolvimento positivo para que os funcionários compreendam que o controle é algo importante.

Neste ponto surge um novo fator. Em diversos hospitais não existe uma política clara de investimento no desenvolvimento de pessoas com a competência para aproximar os profissionais da saúde dos sistemas (PAPA FILHO; POLITANO; VANALLE, 2007). Assim, é importante também que o hospital se preocupe em preparar os facilitadores que irão realizar os treinamentos, para que estes tenham a capacidade de criar uma integração entre profissionais da saúde e o sistema.

É importante que na fase de implementação do sistema sejam realizados treinamentos com os funcionários e, posteriormente, na manutenção do sistema devem ocorrer treinamentos de fixação constantemente, para reafirmar os pontos importantes e motivar os funcionários.

Conforme Rocha (2004), tanto a equipe de implementação quanto os funcionários que usam as informações do sistema precisam ser treinados. Para que as pessoas realmente se integrem ao processo de implementação do sistema 
de custos é necessário que entendam exatamente como acontece o levantamento das informações e o objetivo do sistema.

Barros e Freitas (2005), em seu estudo realizado em quatro hospitais, observaram que equipes que foram treinadas tiveram boa aceitabilidade aos sistemas em três destes hospitais. Contudo, no hospital que houve dificuldades apesar do treinamento, havia também um problema de baixo nível de informatização, o que desmotivou a aceitação do sistema.

\subsection{Utilização de Software}

$\mathrm{O}$ aporte financeiro necessário para a implementação de um sistema de custos é um fator que influencia na escolha e na decisão, principalmente nos hospitais públicos. Contudo, os métodos de custeio geralmente necessitam da utilização de um software para uma melhor aplicação e desenvolvimento do sistema. Rodrigues Filho, Xavier e Adriano (2001) na sua abordagem sobre a implantação de um prontuário automatizado identificaram uma série de problemas atrelados aos sistemas que vem sendo superados lentamente nos hospitais brasileiros (e demais países em desenvolvimento) e concluem que esta automação parece ser promissora na melhoria de qualidade, no avanço do conhecimento e na redução de custos.

Almeida e Mello (2004) detectaram que o uso intenso da tecnologia pelos profissionais da saúde tem aumentado nos últimos anos. Um fator importante para isto é a substituição das gerações de profissionais mais antigas por novas, cuja formação profissional privilegia a tecnologia de modo que a familiaridade com a tecnologia tende a facilitar o processo de implementação do sistema, como visto no estudo de Barros e Freitas (2005), onde o baixo nível de informatização (tecnologia) desmotivou o uso do sistema.

\subsection{Inexistência de Protocolos}

Um importante desafio para a gestão é controlar e reduzir os custos, e ao mesmo tempo manter, ou melhorar, o padrão de qualidade de atendimento hospitalar. A busca por qualidade, custos e efetividade, leva médicos, administradores e planos de saúde ao desenvolvimento de novos métodos de controle. Um destes métodos consiste na implementação de protocolos (TORRES, 2006). Estes protocolos são padronizações de procedimentos médicos, indicadores de rotinas que devem ser adotadas para com o paciente. A equipe médica define a técnica usada no procedimento, os exames diagnósticos pré e pós-operatórios, os materiais e medicamentos necessários, o tipo de internação, e a quantidade de procedimentos ideais. No entanto, há uma dependência da reação do paciente ao tratamento que 
independe das ações realizadas, pois o que em um paciente proporciona uma melhora significativa, em outro pode não ter um resultado (ROCHA, 2004).

Devido à diversidade na formação de cada médico e às particularidades na maneira de diagnosticar, é difícil que um protocolo definitivo seja seguido. Neste caso, o protocolo surge como um roteiro, que não é seguido obrigatoriamente, mas que deve ser analisado para a determinação de uma conduta de atendimento, de modo que o resultado do tratamento seja otimizado. Os hospitais que conseguem padronizar os procedimentos através de protocolos médicos têm maior facilidade de custear seus serviços (SARAIVA, 2001).

Em seu estudo, Torres (2006) conclui que a utilização de protocolos diminui os custos diretos, os quais estão intimamente relacionados aos centros de custos de responsabilidade médica. Como os médicos são responsáveis por $75 \%$ dos custos da organização, presume-se que seja possível a diminuição de parte dos demais custos também. Uma evidência disto é o estudo de Lagioia et al (2008), os quais perceberam que, mesmo em um hospital universitário que possuía como particularidade a escassez de mão-de-obra, a escassez de recursos e a localização em uma região social e economicamente desfavorável, foi possível reduzir custos e atingir de forma mais satisfatória os objetivos do setor analisado.

\section{O Caso Abordado}

O hospital escolhido - especializado em Traumatologia e Cirurgia do Trauma - recebe pacientes com traumatismos, oriundos da região da Grande Porto Alegre, Estado do Rio Grande do Sul, e do sul do Estado de Santa Catarina. Possui 281 leitos, 34 consultórios, 1.026 funcionários e uma área total de 18.325 $\mathrm{m}^{2}$. Já, o setor de ortopedia do hospital apresenta 123 leitos, possui uma média mensal de 220 internações, 174 cirurgias, 1.716 consultas ambulatoriais e 4.880 consultas emergenciais. Vale destacar que $100 \%$ dos serviços são prestados ao Sistema Único de Saúde (SUS).

O primeiro passo da pesquisa consiste na determinação do perfil dos funcionários do setor, no qual se verificou a função exercida. O quadro de funcionários é composto por profissionais com nível superior (25 médicos, 1 fisioterapeuta e 6 enfermeiros) e por profissionais com nível técnico (10 técnicos gessistas e 24 auxiliares ou técnicos em enfermagem). Além disso, a amostra que participa como respondente é condizente com o número total de funcionários do setor, uma vez que se mantém a proporcionalidade entre funcionários com nível superior e funcionários de nível técnico. Percebe-se, também, que todos os funcionários apresentam formação na área da saúde e a maioria dos funcionários (50) é formada há mais de cinco anos, dos quais 34 possuem mais de cinco anos de carreira neste hospital. Além disso, a maioria (55) cumpre mais de 30 horas 
semanais no hospital. Por fim, questionou-se os respondentes sobre a experiência na participação em algum cargo administrativo, onde somente três responderam ter alguma experiência em funções administrativas.

Com base nestas características, conclui-se que os profissionais da amostra estão aptos para a participação na pesquisa, uma vez que o equilíbrio entre a proporção entre nível técnico e superior é condizente e a experiência dos profissionais traduz o conhecimento das rotinas e detalhes do hospital. Além disso, percebe-se que a experiência administrativa no grupo é pequena, o que pode dificultar no processo de implementação de um sistema de custos.

\section{A Pesquisa}

Nesta etapa da pesquisa realizou-se o levantamento da opinião dos funcionários por meio de entrevistas e por meio da aplicação do questionário. Deste modo, este capítulo apresenta as respostas das questões vinculadas ao conhecimento, uso e envolvimento dos funcionários com o sistema de custos do hospital.

\subsection{Conhecimento sobre o Sistema de Custos do Hospital}

Neste primeiro ponto da pesquisa buscou-se estabelecer qual o nível de conhecimentos dos funcionários em relação ao sistema de custos. A pergunta e as respectivas respostas estão dispostas na Tabela 1.

Tabela 1: Conhecimento sobre o sistema de custos do hospital

\begin{tabular}{|c|c|c|c|c|}
\hline \multirow{2}{*}{$\begin{array}{l}\text { O que você sabe sobre o sistema de custos que é aplicado no } \\
\text { hospital? }\end{array}$} & \multicolumn{2}{|c|}{ Nível superior } & \multicolumn{2}{|c|}{ Nível técnico } \\
\hline & Quant. & $\%$ & Quant. & $\%$ \\
\hline Não conheço o sistema de custos & 23 & 85,19 & 21 & 56,76 \\
\hline Conheco o sistema, mas não participo do processo & 4 & 14,81 & 13 & 35,14 \\
\hline Conheço o sistema e todas as suas implicações técnicas & - & - & 1 & 2,70 \\
\hline $\begin{array}{l}\text { Conheço o sistema e participo do processo dando informações } \\
\text { quando questionado }\end{array}$ & - & - & 1 & 2,70 \\
\hline $\begin{array}{l}\text { Conheço o sistema e participo de reuniões para discutir a } \\
\text { manutenção e os resultados dos custos hospitalares }\end{array}$ & - & - & 1 & 2,70 \\
\hline Total & 27 & 100,00 & 37 & 100,00 \\
\hline
\end{tabular}

Fonte: Dados da pesqusia.

Com base nestas respostas, percebe-se que a maioria dos funcionários não conhece o sistema de custos. Somente duas pessoas (do nível técnico) afirmaram estar envolvidas efetivamente no uso e manutenção do sistema. Dentre os que não conhecem o sistema encontra-se, inclusive, o diretor do setor. Com este cenário o funcionamento do sistema é prejudicado uma vez que o nível de comprometimento com algo desconhecido tende a ser baixo. 


\subsection{Informações sobre o Custo de cada Atendimento Realizado}

Nesta segunda questão buscou-se identificar o grau de conhecimento dos funcionários com relação aos custos dos procedimentos realizados no setor. Conforme as respostas apresentadas na Tabela 2 a maioria dos profissionais alegou que não recebe informações, ou recebe informações parciais sobre os custos dos atendimentos realizados. Somente dois dos funcionários, de nível técnico, recebem informações destes custos. O gestor do setor de ortopedia também não recebe informações dos custos dos serviços prestados no seu setor. Contudo, segundo o gestor do setor de contabilidade, estas informações são disponibilizadas, por meio de um demonstrativo mensal do hospital a todos os gestores de setor.

Tabela 2: Informação sobre o custo dos atendimentos realizados

\begin{tabular}{lcccc}
\hline \multirow{2}{*}{ Você sabe o custo dos atendimentos realizados na sua unidade? } & \multicolumn{2}{c}{ Nível superior } & \multicolumn{2}{c}{ Nível técnico } \\
\cline { 2 - 5 } & Quant. & $\%$ & Quant. & $\%$ \\
\hline Não tenho informações sobre os atendimentos da unidade & 18 & 64,29 & 23 & 62,16 \\
Sim, tenho informações, mas são parciais & 5 & 17,86 & 7 & 18,92 \\
Não sei responder, melhor não opinar & 1 & 3,57 & 7 & 18,92 \\
Sim, eu tenho informações sobre os atendimentos da unidade em & 2 & 7,14 & - & - \\
que trabalho & 2 & 7,14 & - & - \\
Não, e acho esta informação sem importância & 28 & 100,00 & 37 & 100,00 \\
\hline Total & & & & \\
\hline
\end{tabular}

Fonte: Dados da pesqusia..

Este fato levanta indícios de dificuldades de comunicação entre o setor da administração e o setor de ortopedia e fortalece a observação de Rocha (2004) sobre a divisão entre as áreas administrativa e da saúde.

\subsection{Participação no Processo de Implementação do Sistema de Custos}

Com aplicação desta questão buscou-se identificar de que forma os profissionais do setor de ortopedia participam do processo de implementação do sistema de custos do hospital, cujas respostas se encontram na Tabela 3. 


\section{Tabela 3: Participação no processo de implementação do sistema de custos}

\begin{tabular}{lcccr}
\hline \multirow{2}{*}{$\begin{array}{l}\text { De que forma você participa do processo de implementação do } \\
\text { sistema de custos? }\end{array}$} & \multicolumn{2}{c}{ Nível superior } & \multicolumn{2}{c}{ Nível técnico } \\
\cline { 2 - 5 } & Quant. & $\%$ & Quant. & $\%$ \\
\hline $\begin{array}{l}\text { Fornecendo informações quanto à quantidade gasta em minhas } \\
\text { atividades com materiais, para a equipe de custos }\end{array}$ & 10 & 33,33 & 13 & 52,00 \\
$\begin{array}{l}\text { De outra forma } \\
\text { Utilizando protocolos de procedimentos que são referenciados }\end{array}$ & 9 & 30,00 & 6 & 24,00 \\
pelo hospital & 11 & 36,67 & 1 & 4,00 \\
Analisando os resultados de minha unidade de custos & - & - & 5 & 20,00 \\
Participando de treinamentos de sistema de custos & - & - & - & - \\
\hline Total & 30 & 100,00 & 25 & 100,00
\end{tabular}

Fonte: Dados da pesqusia.

Observa-se que os profissionais com nível superior, quando comparados com profissionais de nível técnico, responderam em maior percentual à utilização de protocolos de procedimentos que são referenciados pelo hospital. Outro aspecto importante é que somente cinco pessoas do nível técnico analisam os custos da unidade. Contudo, o principal fator consiste na não participação dos funcionário nos treinamentos, inclusive o diretor do setor.

\subsection{Importância das Informações}

Na quarta questão buscou-se determinar o grau de importância atribuído às informações de custos pelos funcionários, conforme a Tabela 4.

Apesar de ter sido constatado nas questões anteriores que o nível de conhecimento do sistema no hospital é baixo, que a maioria dos funcionários não conhece o custo dos atendimentos do setor e que a participação no processo de implementação foi praticamente inexistente, a maioria dos funcionários acredita que as informações são importantes e que estas auxiliam na melhoria da gestão no hospital. O fato de $19 \%$ dos respondentes acreditarem que as informações são importantes para o setor administrativo do hospital pode indicar que estes funcionários consideram o sistema um problema do setor administrativo o que possivelmente significará baixo grau de comprometimento.

Tabela 4: Importância das informações de custo

\begin{tabular}{|c|c|c|c|c|}
\hline \multirow{2}{*}{ Qual a importância das informações de custo para você? } & \multicolumn{2}{|c|}{ Nível superior } & \multicolumn{2}{|c|}{ Nível técnico } \\
\hline & Quant. & $\%$ & Quant. & $\%$ \\
\hline São importantes, pois ajudam a melhorar a gestão hospitalar & 23 & 76,67 & 24 & 63,16 \\
\hline É bom para o setor administrativo do hospital & 4 & 13,33 & 9 & 23,68 \\
\hline Não sei responder, melhor não opinar & 3 & 10,00 & 4 & 10,53 \\
\hline Não ajuda no meu trabalho & - & - & 1 & 2,63 \\
\hline Não tem importância & - & - & - & - \\
\hline Total & 30 & 100,00 & 38 & 100,00 \\
\hline
\end{tabular}

Fonte: Dados da pesqusia. 
Nos pontos posteriores do questionário levantou-se a opinião dos funcionários sobre fatores que dificultam ou que poderiam facilitar a implementação do sistema de custos.

\subsection{Atitudes que Facilitam a Colaboração dos Funcionários}

Primeiramente questionou-se os funcionários sobre quais as atitudes por parte do hospital (administração) facilitariam a colaboração no processo de implementação do sistema de custos.

Como pode-se observar nas respostas apresentadas na Tabela 5, a opção assinalada como principal atitude para promover a colaboração dos funcionários foi "a apresentação dos resultados e dos relatórios mensais das unidades do hospital". Como a segunda atitude mais importante foi considerada "realização de treinamentos". Percebeu-se ainda que a opção "treinamentos por pessoas do próprio hospital" foi assinalada por alguns funcionários. Desta maneira, percebese que os funcionários esperam que o hospital os envolva no processo de implementação do sistema com treinamentos constantes e, também, esperam ter um feedback do funcionamento do sistema e das informações obtidas. Além disso, percebe-se um desejo de que o treinamento seja realizado por pessoas do hospital, o que comprova a falta dos profissionais internos habilitados para se tornarem os facilitadores entre profissionais da saúde e o sistema.

Tabela 5: Atitudes que facilitam a colaboração dos funcionários

\begin{tabular}{lcccc}
\hline Quais atitudes do hosp ital que facilitam a colaboração dos & \multicolumn{2}{c}{ Nível superior } & \multicolumn{2}{c}{ Nível técnico } \\
\cline { 2 - 5 } funcionários no processo de implementação? & Quant. & $\%$ & Quant. & \multicolumn{1}{c}{$\%$} \\
\hline Apresentação dos resultados mensais das unidades do hospital & 14 & 41,18 & 16 & 40,00 \\
Treinamento constante dos funcionários & 10 & 29,41 & 12 & 30,00 \\
$\begin{array}{l}\text { Treinamento realizado por profissionais da empresa e não por } \\
\text { consultores externos }\end{array}$ & 5 & 14,70 & 3 & 7,50 \\
Uma estrutura da organização hospitalar mais descentralizada & 1 & 2,94 & 4 & 10,00 \\
$\begin{array}{l}\text { Participação financ eira dos funcionários nos lucros que o } \\
\text { hospital tiver com a redução de despesas referentes à aplicação } \\
\text { do novo sistema }\end{array}$ & - & - & 4 & 10,00 \\
$\begin{array}{l}\text { Funcionários com uma carga horária elevada, se possível com } \\
\text { dedicação exclusiva ao hospital }\end{array}$ & 3 & & & \\
Outra atitude & 1 & 2,94 & - & - \\
\hline Total & 34 & 100,00 & 40 & 100,00 \\
\hline
\end{tabular}

Fonte: Dados da pesqusia. 
5.6 Fatores Culturais que Dificultam a Implementação

Nesta questão questionou-se quais os principais fatores culturais que os funcionários julgam dificultar o processo de implementação do sistema de custos. As respostas obtidas são apresentadas na Tabela 6.

Tabela 6: Fatores culturais que dificultam a implementação

\begin{tabular}{|c|c|c|c|c|}
\hline \multirow{2}{*}{$\begin{array}{l}\text { Quais os principais fatores culturais que dificultam o processo de } \\
\text { implementacãa do sistema de custos? }\end{array}$} & \multicolumn{2}{|c|}{ Nível superior } & \multicolumn{2}{|c|}{ Nível técnico } \\
\hline & Quant. & $\%$ & Quant. & $\%$ \\
\hline A resistência à mudança da rotina diária dentro do hospital & 13 & 31,71 & 23 & 43,40 \\
\hline Receio de que se considere apenas a quantidade e não a qualidade & 19 & 46,34 & 14 & 26,41 \\
\hline $\begin{array}{l}\text { A cultura de que o hospital tem que se preocupar somente em } \\
\text { curar os pacientes, não importando o quanto isto vai custar para a } \\
\text { organização }\end{array}$ & 6 & 14,63 & 9 & 16,98 \\
\hline $\begin{array}{l}\text { Receio de que a administração hospitalar verifique que a sua } \\
\text { produção dentro do hospital está muito baixa }\end{array}$ & 1 & 2,44 & 5 & 9,43 \\
\hline Corporativismo dos profissionais da saúde & 2 & 4,88 & 1 & 1,89 \\
\hline Outro fator & - & - & 1 & 1,89 \\
\hline Total & 41 & 100,00 & 53 & 100,00 \\
\hline
\end{tabular}

Fonte: Dados da pesqusia.

De acordo com as respostas, verifica-se que os profissionais têm dificuldade em aceitar a mudança de sua rotina diária, principalmente pelo fato de a maioria trabalhar na instituição há muitos anos. Este resultado confirma a análise de Cogan (1994) que afirma que a resistência cultural decorre da mudança, uma vez que os profissionais sentem o seu cargo ameaçado e, algumas vezes, boicotam a implementação do sistema de custos. Na entrevista com o gestor contábil do hospital este afirmou que a resistência cultural é a principal barreira cultural existente no hospital, segundo a sua visão. Por outro lado, pode existir um sentimento de que o tempo utilizado para informar os dados ao sistema seja um tempo "desperdiçado", pois não é utilizado no tratamento aos pacientes.

O receio de que os trabalhos dos profissionais ou da unidade sejam avaliados, mais quantitativamente que qualitativamente, também é apontado na pesquisa, pois o sistema de custos é assimilado pelas pessoas como um índice quantitativo. Um exemplo é o receio de que um funcionário que atender um maior número de pacientes em menor tempo possa ser mais valorizado que outro profissional que atender menor número de pacientes no mesmo tempo, sem que seja considerada a qualidade, e até mesmo a complexidade do atendimento.

A questão que aborda a "cultura do hospital em se preocupar somente em curar os pacientes sem se importar com os custos da instituição", citada por Rocha 
(2004) como uma das principais dificuldades culturais - tendo em vista que os profissionais da saúde costumam tomar suas decisões somente com o intuito de salvar a vida do paciente - não se confirmou como a principal barreira cultural na visão dos profissionais da saúde participantes neste estudo.

A questão sobre o receio de verificar que a produção individual é baixa não foi muito assinalada, apesar de os profissionais terem receio de serem analisados sob uma ótica que privilegie a quantidade e não a qualidade.

O questionamento sobre corporativismo dos profissionais da saúde foi o menos assinalado, apesar de algumas profissões da área da saúde, principalmente a médica e a enfermagem, exercerem certo corporativismo.

\subsection{Implementação de Protocolos de Atendimento}

A sétima questão aborda a utilização do protocolo de padronização das atividades realizadas pelos profissionais de saúde que é importante instrumento para o controle de custos. Neste sentido, questionou-se os funcionários sobre quais seriam as principais dificuldades na implementação dos protocolos, cujas respostas são apresentadas na Tabela 7 .

A divisão existente entre a área administrativa e a área da saúde foi apontada como o principal empecilho para a instalação de protocolos. Isto, possivelmente, porque os protocolos precisam ser analisados pelas duas áreas, levando-se em conta os custos e as características de cada patologia tratada.

A resistência cultural dos profissionais confirma as respostas da questão anterior, onde a resistência a mudanças na rotina também foi assinalada. Os profissionais estão acostumados com as rotinas diárias no cuidado aos pacientes segundo o seu próprio roteiro de tratamento.

Tabela 7: Principais dificuldades para a implementação dos protocolos

\begin{tabular}{lcccc}
\hline \multirow{2}{*}{$\begin{array}{l}\text { Quais as principais dificuldades para implementação dos } \\
\text { protocolos? }\end{array}$} & \multicolumn{2}{c}{ Nível superior } & \multicolumn{2}{c}{ Nível técnico } \\
\cline { 2 - 5 } & Quant. & $\%$ & Quant. & $\%$ \\
\hline Divisão existente entre a área da saúde e a área administrativa & 15 & 36,59 & 15 & 33,33 \\
Resistência cultural dos profissionais da saúde & 8 & 19,51 & 16 & 35,56 \\
Protocolos mal formulados, o que pode levar risco ao paciente & 11 & 26,83 & 9 & 20,00 \\
Diferente formação dos profissionais da saúde & 7 & 17,07 & 2 & 4,44 \\
De outra forma & - & - & 3 & 6,67 \\
\hline Total & 41 & 100,00 & 45 & 100,00 \\
\hline
\end{tabular}

Fonte: Dados da pesqusia.

A má formulação dos protocolos, que levam risco ao paciente, também foi 
assinalada. Wolf et al. (1999) citam que esta é a principal dificuldade na implementação de protocolos. Parte da má formulação pode ser decorrente das dificuldades derivadas da divisão existente entre a área administrativa e a área da saúde, e pela própria essência dos serviços, que são difíceis de serem padronizados.

A diferente formação dos profissionais foi a quarta alternativa mais assinalada. Apesar de ser citada por Torres (2006) como uma das principais dificuldades para implementar protocolos, os profissionais do hospital não consideraram esse fato tão relevante.

Os profissionais de nível superior avaliam que a divisão existente entre a área da saúde e a administrativa é a principal dificuldade da implementação de protocolos, enquanto os profissionais de nível técnico consideraram mais importante a resistência cultural dos profissionais de saúde. O gestor do setor de ortopedia também considerou estes dois como os principais fatores. Já o gestor contábil não opinou sobre as principais dificuldades, mas confirmou serem importantes os protocolos para a padronização dos procedimentos, pois facilitam o controle dos custos.

\subsection{Estrutura Administrativa}

$\mathrm{Na}$ Tabela 8 apresentam-se as respostas da oitava questão que levantou a opinião dos funcionários do setor sobre os fatores da estrutura administrativa do hospital que mais dificultam o processo de implementação do sistema de custos.

Observando-se as respostas da Tabela 8 percebe-se que o fator que exerce maior influência na implementação do sistema é a divisão entre as áreas administrativa e da saúde. Esta divisão pode explicar parcialmente as respostas anteriores, tais como o não recebimento dos resultados mensais pelo setor de saúde, a resistência à mudança pelos profissionais da saúde - que pode ser atribuída à administração também, pois esta também não compartilha informações de custos. Além das questões que este fator já foi apontado diretamente.

Por outro lado, a não-participação da direção na implementação não foi muito assinalada. Isso, possivelmente, pode ser explicado pelo fato de ser uma instituição pública e os principais diretores terem cargos políticos, o que pode justificar, sob a ótica dos funcionários, pouca importância às figuras diretivas. 


\section{Tabela 8: Fatores da estrutura administrativa}

\begin{tabular}{lccccc}
\hline Quais os fatores na estrutura administrativa do hospital que mais & \multicolumn{2}{c}{ Nível superior } & \multicolumn{2}{c}{ Nível técnico } \\
\cline { 2 - 6 } dificultam o processo de implementação de um sistema de custos? & Quant. & $\%$ & Quant. & $\%$ \\
\hline Divisão existente entre as áreas administrativa e da saúde & 19 & 45,24 & 21 & 47,73 \\
Estrutura centralizada da administração hospitalar & 9 & 21,43 & 8 & 18,18 \\
Falta de tecnologia. Ex: computadores, software & 7 & 16,67 & 6 & 13,64 \\
Não-participação da direção na implementação & 3 & 7,14 & 3 & 6,82 \\
Muitos profissionais com pequena carga horária no hospital, o & 4 & 9,52 & 2 & 4,54 \\
que diminui o comprometimento com a organização & & & 4 & 9,09 \\
Outro Fator & - & - & 100,00 & 44 & 100,00 \\
\hline Total & 42 & & &
\end{tabular}

Fonte: Dados da pesqusia.

\subsection{Grau de dificuldade na implementação de um sistema de custos}

Por fim, foram determinadas as dez principais dificuldades para implementação de um sistema de custos, adaptadas do estudo realizado por Rocha (2004). Nesta questão solicitou-se que os respondentes ordenassem as dificuldades, atribuindo-lhes números de 1 a 10 . Foi assinalado com o número 1 o item considerado de maior dificuldade e, assim crescentemente até o considerado de menor dificuldade que foi marcado com o número 10. As respostas foram separadas entre funcionários de nível técnico $(\mathrm{T})$ e superior $(\mathrm{S})$, conforme apresentado no Quadro 1.

\section{Quadro 1:Fatores que dificultam a implementação}

\begin{tabular}{|c|c|c|c|c|c|c|c|c|c|c|c|c|c|c|c|c|c|c|c|c|}
\hline \multirow{4}{*}{ Variáveis que geram dificuldades } & \multicolumn{20}{|c|}{ Ordem de dificuldade } \\
\hline & \multicolumn{14}{|c|}{ Maior dificuldade - } & \multicolumn{6}{|c|}{ Menor dificuldade } \\
\hline & \multicolumn{2}{|r|}{1} & \multicolumn{2}{|c|}{2} & \multicolumn{2}{|c|}{3} & \multicolumn{2}{|c|}{4} & \multicolumn{2}{|c|}{5} & \multicolumn{2}{|c|}{6} & \multicolumn{2}{|c|}{7} & \multicolumn{2}{|c|}{8} & \multicolumn{2}{|c|}{9} & \multicolumn{2}{|c|}{10} \\
\hline & $\mathrm{S}$ & $\mathrm{T}$ & S & $\mathrm{T}$ & S & $\mathrm{T}$ & $\mathrm{S}$ & $\mathrm{T}$ & $S$ & $\mathrm{~T}$ & S & $\mathrm{T}$ & $\mathrm{S}$ & $\mathrm{T}$ & $\mathrm{S}$ & $\mathrm{T}$ & $S$ & $\mathrm{~T}$ & $\mathrm{~S}$ & $\mathrm{~T}$ \\
\hline Falta de planejamento do projeto & 6 & 8 & 9 & 3 & 0 & 1 & 2 & 4 & 4 & 4 & 0 & 1 & 0 & 0 & 2 & 1 & 0 & 2 & 2 & 0 \\
\hline Falta de conhecimento $\mathrm{p} /$ utilizar inform & 4 & 7 & 2 & 6 & 6 & 1 & 1 & 1 & 0 & 3 & 4 & 2 & 4 & 1 & 3 & 1 & 1 & 0 & 0 & 2 \\
\hline $\begin{array}{l}\text { Divisão entre as áreas da saúde e } \\
\text { administrativa }\end{array}$ & 5 & 3 & 3 & 2 & 2 & 2 & 6 & 3 & 4 & 4 & 1 & 3 & 3 & 2 & 0 & 1 & 0 & 3 & 1 & 1 \\
\hline Resistência cultural & 4 & 2 & 5 & 5 & 2 & 3 & 0 & 3 & 2 & 3 & 1 & 0 & 2 & 3 & 1 & 2 & 3 & 3 & 5 & 0 \\
\hline Falta de treinamento & 0 & 1 & 0 & 3 & 3 & 6 & 2 & 5 & 0 & 0 & 3 & 2 & 8 & 5 & 2 & 1 & 0 & 0 & 1 & 1 \\
\hline Estrutura administrativa & 0 & 3 & 2 & 3 & 3 & 0 & 5 & 0 & 4 & 1 & 6 & 7 & 2 & 1 & 3 & 6 & 0 & 3 & 0 & 0 \\
\hline Inexistência de protocolos & 3 & 0 & 4 & 0 & 2 & 3 & 2 & 2 & 3 & 0 & 1 & 2 & 4 & 6 & 2 & 2 & 3 & 5 & 1 & 4 \\
\hline Treinamento por consultores & 0 & 0 & 0 & 2 & 4 & 3 & 2 & 2 & 0 & 5 & 1 & 2 & 0 & 3 & 6 & 6 & 7 & 1 & 5 & 0 \\
\hline Não-participação da direção & 3 & 0 & 0 & 0 & 1 & 5 & 4 & 4 & 1 & 1 & 4 & 3 & 2 & 2 & 4 & 3 & 3 & 1 & 3 & 5 \\
\hline Utilização de software & 0 & 0 & 0 & 0 & 2 & 0 & 1 & 0 & 1 & 3 & 4 & 2 & 0 & 1 & 2 & 1 & 8 & 6 & 7 & | \\
\hline
\end{tabular}

Fonte: Dados da pesqusia. 
Posteriormente, como forma de facilitar a análise, estes dados foram convertidos em um fator que permite a visualização das principais dificuldades numa ordem de importância, segundo o ponto de vista dos funcionários. Esta conversão se deu pela multiplicação da quantidade de "escolhas" em cada posição na linha de dificuldade, por um peso. Para isto, determinou-se o peso 10 para a variável considerada de maior dificuldade, e 1 para a variável considerada de menor dificuldade.

Com a conversão de todas as variáveis obtém-se um grau de dificuldade que permite ordená-las por dificuldade, conforme apresentado nas Figuras 1 (resultado dos funcionários com nível superior) e 2 (resultados dos funcionários de nível técnico).

Figura 1: Grau de dificuldade, segundo os funcionários com nível superior

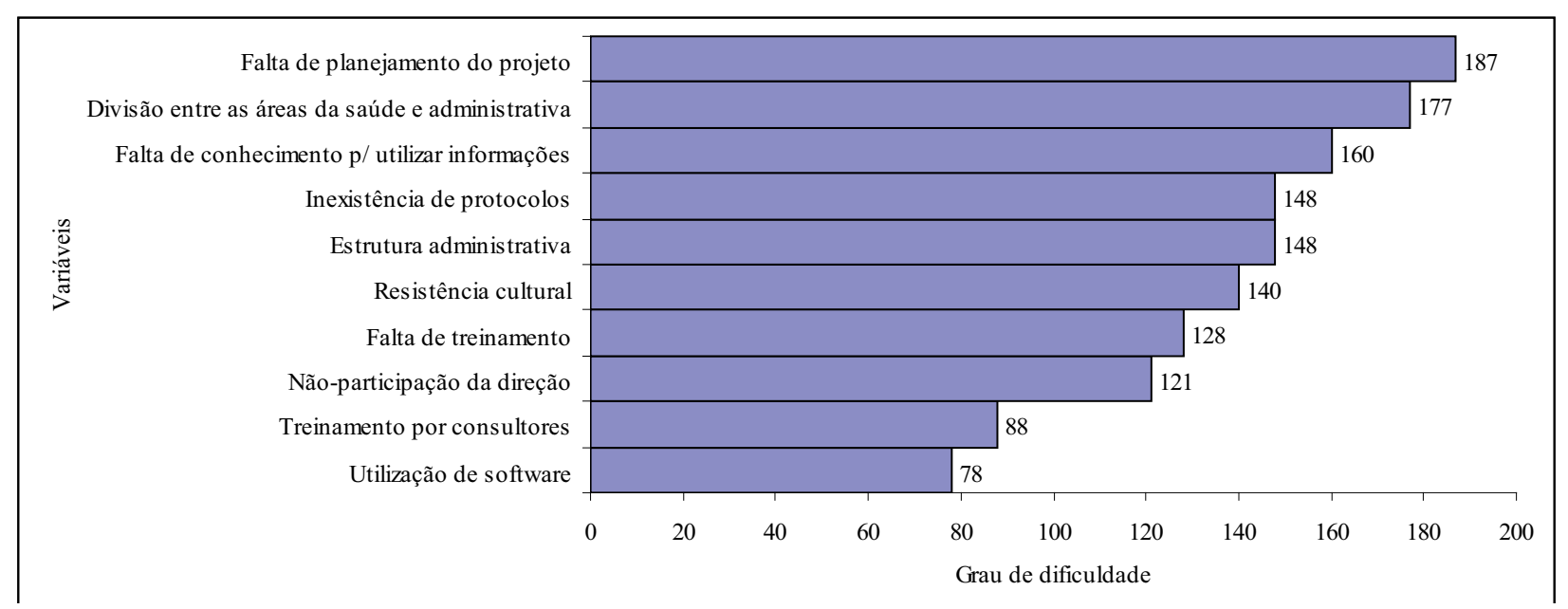

Fonte: Dados da pesqusia.

Para os funcionários que possuem nível superior as duas variáveis que mais dificultam a implementação do sistema de custos são a "falta de planejamento do projeto" e a "divisão entre as áreas da saúde e administrativa". Já, a "utilização de softwares" e o "treinamento realizado por consultores" são as variáveis consideradas menos importantes.

Já para os funcionários de nível técnico a variável de maior importância é a "falta de planejamento do projeto" e em segundo lugar a "falta de conhecimento para utilizar as informações". Contudo, como variáveis de menor importância foram assinaladas a "utilização de softwares" e a "inexistência de protocolos". 
Figura 2: Grau de dificuldade, segundo os funcionários de nível técnico

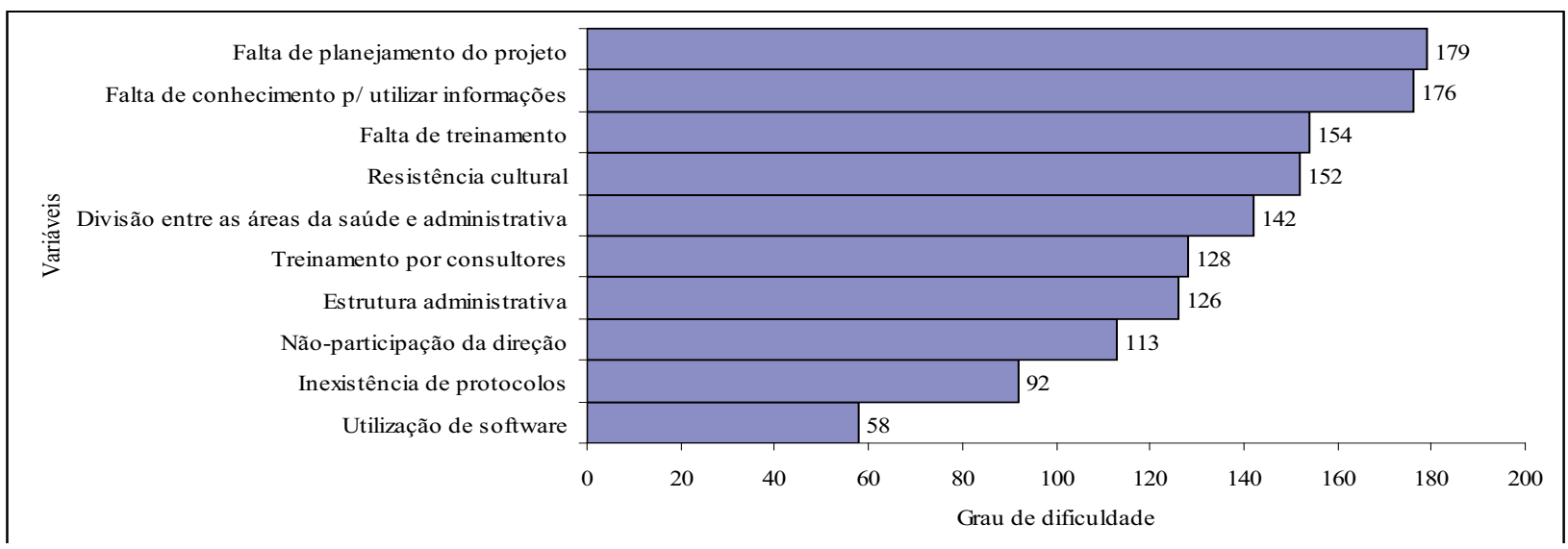

Fonte: Dados da pesqusia.

Numa análise conjunta dos resultados, pode-se inferir que a falta de planejamento, talvez, seja realmente o principal problema envolvido no processo de implementação de um sistema de gestão de custos, uma vez que existem diversas outras variáveis atreladas. Como o planejamento é o primeiro passo da implementação, se este for executado, tem-se um maior envolvimento dos profissionais de ambas as áreas - administração e saúde - aos programas de treinamento - que possibilitam a aquisição de conhecimento de como utilizar as informações - e a criação de protocolos por meio da utilização de softwares.

\section{Entrevista com os Gestores}

$\mathrm{Na}$ última etapa da pesquisa foram entrevistados os gestores dos setores administrativo e de ortopedia. O diretor do setor de ortopedia é médico e está no cargo há dois anos. Já o assessor administrativo do setor de controladoria é formado em Ciências Contábeis e trabalha há sete anos no hospital.

Segundo o gestor contábil, o método de custeio aplicado no hospital é o Absorção, implantado em 1997. Por outro lado, o gestor do setor de ortopedia não tem nenhum conhecimento sobre as especificidades técnicas do método de custeio. Contudo, ambos concordam que o sistema de custos é importante para o hospital.

Quanto à participação dos profissionais de saúde, o gestor contábil enfatizou que apenas os gestores de unidades têm acesso aos resultados pelo sistema de custos e que estes utilizam tais informações como ferramentas para melhor gerenciar a sua unidade. Mesmo assim, ele crê que as informações não são utilizadas na sua totalidade por falta de conhecimento das pessoas da área da saúde. Todavia, destacou a importância de se saber quanto custa o serviço e o aumento na transparência dos atos da administração. Já, o gestor do setor de ortopedia relatou que não recebe os relatórios e não tem acesso às informações do sistema, 
e somente recebe orientações esporádicas para a redução de custos.

Quanto à interferência da cultura no processo de implementação de um sistema de custos, o gestor contábil enfatizou a questão da resistência à mudança dos profissionais da saúde. No entanto, o gestor da ortopedia relatou que a principal dificuldade é a divisão existente entre a área administrativa e a área da saúde.

No quesito "utilização de protocolos", o gestor da ortopedia relata que estes não existem e que a sua implementação é importante para maior controle no setor. Segundo ele, no caso de implementação de protocolos, ai sim a cultura da organização representaria a maior dificuldade, pois não há uma cultura de utilização de protocolos no hospital. O gestor contábil, também, afirma que os protocolos são importantes, pois uma maior padronização dos serviços facilitaria o mapeamento dos custos.

Ambos concordaram, ainda, que uma estrutura administrativa mais descentralizada, que proporcione mais autonomia aos setores, poderia facilitar o processo, principalmente por se tratar de um hospital público.

\section{Considerações Finais}

O presente estudo objetivou identificar os fatores que influenciam o processo de implementação de um sistema de gestão de custos em um hospital público do Rio Grande do Sul. Alguns fatores que dificultam os processos de implementação de sistemas de custos foram levantados por meio de pesquisa bibliográfica e serviram de base para o estabelecimento do roteiro de entrevistas. Os fatores identificados foram: falta de planejamento do projeto; falta de conhecimento para utilizar as informações; divisão existente entre a área da saúde e a administrativa; resistência cultural; falta de treinamento; estrutura administrativa; inexistência de protocolos; não-participação da direção; treinamento por consultores; e, utilização de software. Percebeu-se que alguns fatores apresentados por outros autores não se confirmaram neste caso, e outros que se apresentaram como principais em outros estudos, neste estudo se apresentaram como secundários.

De forma geral, as opiniões dos profissionais de nível técnico e de nível superior do setor de ortopedia não apresentaram grandes divergências nas opiniões, uma vez que quando não eram iguais, pelo menos apresentavam semelhanças.

Pode-se concluir que a maior dificuldade está relacionada à divisão entre o setor de ortopedia e o setor administrativo. Isto porque além desta dificuldade ser apontada diretamente como importante, diversas outras que foram apontadas também derivam deste fator. Esta dificuldade se confirma nas entrevistas com os gestores quando estes divergem sobre o recebimento ou não das informações sobre custos. Além disso, enquanto que a área administrativa identifica, para a área de saúde, a "falta de conhecimento para utilizar as informações" e a "resistência 
cultural" como as principais dificuldades, inversamente, os profissionais da saúde consideram a "falta de planejamento do projeto" e a "falta de conhecimento para utilizar as informações" como as principais dificuldades do processo de implementação de um sistema de custos.

Como os profissionais da saúde se mostraram interessados em participar do processo, pode-se concluir que o fornecimento de relatórios de custos para as unidades e a realização de treinamentos para o uso do sistema, concomitantemente com o uso das informações, podem ajudar no processo de implementação do sistema de gestão de custos e representar uma possível solução para as principais dificuldades encontradas, e o início para a redução da separação entre ambos os setores.

\section{Referências}

ALMEIDA, G. W.; MELLO, R. C. Uso de novas tecnologias de informação por profissionais da área da saúde na Bahia. Revista de Administração Contemporânea, Rio de Janeiro, v. 8, n. 3, p. 9-27, jul./set. 2004.

ALVES, R. V.; BORGERT, A.; SCHULTZ, C. A. A influência da cultura organizacional na implementação de um sistema de controle de custos em hospitais. In: XXVI Encontro Nacional de Engenharia de Produção. Anais... Fortaleza: Abepro, 2006. CD-ROM.

BARROS, L. A. C da; FREITAS, A. A. F. de. Análise de sistemas de gestão de custos em hospitais públicos. In: XXV Encontro Nacional de Engenharia de Produção. Anais... Porto Alegre: Abepro, 2005. CD-ROM.

BORBA, G. S. de; CUNHA, A. K. M. da; SORDI, D. R.; BORGES, M. V. Saúde Integrada? Um estudo exploratório sobre o uso de Sistemas Integrados de Gestão (ERP) nos hospitais gaúchos. In: XVII Encontro da ANPAD. Anais... Atibaia: Anpad, 2003. CD-ROM.

BRASIL. Lei n ${ }^{\circ} 9.656$, de 03 de junho de 1998. Dispõem sobre os planos e seguros privados de assistência à saúde. Lex: Disponível em: <http://www.planalto.gov. br/ccivil_03/Leis/L9656.htm>. Acesso em: 28 jun. 2008.

CARPINTÉRO, J. N. C. Custos na área da saúde: considerações teóricas. In: VI Congresso Brasileiro de Custos. Anais... .São Paulo: ABC, 1999. CD-ROM.

COGAN, S. Activity based costing (ABC): a poderosa estratégia empresarial. São Paulo: Thomson Pioneira, 1994. 
COSTA, T. C. S.; CARVALHO, J. B. C. Cost accounting applications in Logal Governments: the case of municipal tariff and price setting in the North of Portugal. Revista Contemporânea de Contabilidade. v. 1, n. 7, p. 11-24, jan./jun. 2007.

GIL, A. C. Como elaborar projetos de pesquisa. 3. ed. São Paulo: Atlas, 1992.

LAGIOIA, U. B. T.; FALK, J. A.; RIBEIRO FILHO, J. F.; LIBONATI, J. J. A gestão por processos gera melhoria na qualidade e redução de custos: o caso da unidade de ortopedia e traumatologia do Hospital das Clínicas da Universidade Federal de Pernambuco. Revista de Contabilidade e Finanças. v. 19, n. 48, p. 77 90, setembro/outubro. 2008.

LIMA, C. R. M.; LIMA, C. R. M. A avaliação do custo-eficácia das intervenções em organizações de saúde. Revista de Administração de Empresas, São Paulo, v. 38, n. 2, p. 62-73, abr. /jun. 1998.

PANEGALLI, J. C. Facilitando o processo de planejamento e gestão nas organizações. Revista Contemporânea de Contabilidade, Florianópolis, v. 1, n. 2, p. 11-40, jul./dez. 2004.

PAPA FILHO, S.; POLITANO, P. R.; VANALLE, R. M. A tecnologia da informação hospitalar: estudo de multicasos na implementação de um sistema de registro em clínicas de dor crônica. Interseção, Belo Horizonte, v. 1, n. 1, p. 1-11, out., 2007.

PATTIO, M. L. P. O impacto do sistema ABC no controle organizacional da Casa de Saúde Santa Maria. 2001. 95 f. Dissertação (Mestrado em Engenharia de Produção) - Programa de Pós-Graduação em Engenharia de Produção, Universidade Federal de Santa Catarina, Florianópolis.

POMPERMAYER, C. B. Sistemas de gestão de custos: dificuldades de implementação. Revista FAE, Curitiba, v. 2, n. 3, p. 21-28, set./dez. 1999.

RICHARDSON, R. J. Pesquisa social: métodos e técnicas. 2. ed. São Paulo: Atlas, 1989.

ROCHA, V. L. Dificuldades na implantação de sistemas de custeio em instituições hospitalares: um estudo de caso em um hospital de Florianópolis. 2004. 115f. Dissertação (Mestrado em Engenharia de Produção) - Programa de PósGraduação em Engenharia de Produção, Universidade Federal de Santa Catarina, Florianópolis.

RODRIGUES FILHO, J.; XAVIER, J. C. B.; ADRIANO, A. L. A tecnologia 
da informação na área hospitalar: um caso de implementação de um sistema de registros de pacientes. RAC. v. 5, n. 1, p. 105-120. Jan./Abr. 2001.

SALGUES, L. J. V. et al. A percepção de aspectos culturais da organização: um estudo sob o enfoque do controle organizacional. In: $8^{\circ}$ Seminário em Administração FEA-USP. Anais... Disponível em: < http://www.ead.fea.usp.br/Semead/8semead/ resultado/trabalhosPDF/170. pdf>, 2005. Acesso em: 23 mar. 2007.

SARAIVA, S. Hospital São Mateus cria protocolo de dor torácica. Diário do Nordeste, Fortaleza, 23 mar. 2001. Disponível em: <http://diariodonordeste.globo. com/2001/03/23/ 010046.htm>. Acesso em: 23 mar. 2007.

SELLTIZ, C.; WRIGHTSMAN, L.; COOK, S. Métodos de pesquisas nas relações sociais: delineamentos de pesquisa. São Paulo: EPU, 1987.

TORRES, U. C. T. L. Gestão por processos: o protocolo como ferramenta para a melhoria da qualidade e de redução dos custos nos hospitais universitários. 2006. 274 f. Tese (Doutorado em Administração) - Programa de Pós-Graduação em Administração, Universidade Federal de Pernambuco, Recife.

WINTER, A.; HAUX, R. A three-level graph-based model for the management of hospital information systems. Methods of Information in Medicine, v. 34, p. 378-96, 1994.

WOLF, S.H. et al. Clinical guidelines potential benefits, limitations, and harms or clinical guidelines. BMJ Publishing Group. v. 318, p. 527-530, feb. 1999. 
